\title{
Experiences of nurses implementing evidence-based practice in an acute care setting in Nigeria: $A$ qualitative study
}

Jude N. Ominyi*1, David A. Agom ${ }^{1}$, Chukwuma N. Anyigor ${ }^{2}$, Aaron B. Nwedu ${ }^{3}$, Simon N. Onwe ${ }^{4}$

${ }^{1}$ University of Northampton, United Kingdom

${ }^{2}$ University of Southampton, United Kingdom

${ }^{3}$ University of Salford, United Kingdom

${ }^{4}$ Queen Margaret University, Edinburgh, United Kingdom

Received: June 29, 2019

DOI: $10.5430 /$ cns.v7n4p54
Accepted: September 25, $2019 \quad$ Online Published: October 9, 2019

URL: https://doi.org/10.5430/cns.v7n4p54

\begin{abstract}
Background: Evidence-based practice (EBP) is widely recognised as being relevant in improving nursing care. However, its implementation in nursing practice has been difficult for nurses, particularly in the developing countries. Existing evidence suggests that bureaucracy in organisational governance impacts implementation processes, however, the nature of this effect is not yet explored. Objective: The study examined experiences of nursing staff implementing EBP in a bureaucratic acute care setting in Nigeria.

Methods: A qualitative exploratory design was adopted. A purposive sample of 11 ward managers and 12 staff nurses from a large acute care setting in Nigeria participated in the in-depth, face-to-face interviews. Data was analysed using the thematic analysis approach.

Results: Four key themes emerged: (1) top down managerial approach; (2) nurse and nurse manager relations; (3) managerial prerogatives; (4) managerial autonomy.

Conclusions and implications for practice: The Nigerian bureaucracy limits professional and managerial autonomy that nurses require in driving EBP implementation. Nurse Managers require greater leadership visibility and structural empowerment to create enabling environment for EBP implementation in nursing.
\end{abstract}

Key Words: Nursing management, Evidence-based practice, Nurses, Nurse manager, Qualitative interview, Qualitative thematic analysis, Nigeria

\section{INTRODUCTION}

Evidence-based practice (EBP) is widely recognised as being relevant in improving nursing care. ${ }^{[1-3]}$ While there are numerous definitions of EBP, the most common definition is "the integration of the best research evidence with clinical expertise and the patient's preferences and values". ${ }^{[4]}$ It originated from medical science (evidence-based medicine) and has been drastically expanded, adopted, and adapted by other disciplines within the health care arena. From the perspective nursing scholars, for example, Melnyk and colleagues, ${ }^{[2,3]} \mathrm{EBP}$ entails a problem-solving approach to the delivery of health care that integrates best evidence from studies and patient care data with clinician's expertise and patient's preferences and values. As a broader concept, EBP

*Correspondence: Jude N. Ominyi; Email: judeominyi@yahoo.com; Address: University of Northampton, United Kingdom. 
replaces research utilisation (RU) or the use of research findings in practice ${ }^{[5]}$ Research utilisation refers to the process of critiquing, implementing, and evaluating research findings ${ }^{[2]}$ which is very crucial in clinical decision-making and a critical part of EBP in nursing. ${ }^{[6]}$

The incorporation of EBP into daily care delivery does not only improve patient outcomes but facilitates patient safety. ${ }^{[3,7]}$ Implementation of EBP is currently a priority for healthcare organisations globally due to its cost-efficiency and effectiveness. ${ }^{[8,9]}$ However, available evidence suggests that implementation of EBP is hindered several factors, ${ }^{[10,11]}$ for example, lack of nurses' individual and organisational readiness for EBP, which is further complicated by lack of best evidence in a form that is useful for and easily translated and integrated into practice. ${ }^{[12,13]}$ Consequently, contrary to the expectation that implementation of EBP should be the norm in daily practice, majority of nurses and other clinicians do not consistently engage in EBP. ${ }^{[12,14]}$ There are concerns regarding the inability of healthcare managers to address barriers to implementation of EBP currently experienced in healthcare settings. ${ }^{[5,15]}$

It is suggested that EBP is not prioritised by policymakers and healthcare managers, particularly in several developing countries. ${ }^{[15,16]}$ This might imply that initiatives proposing EBP do not align with organisational goals, mission or vision. Previous studies conducted in some developing countries report cultural barriers to EBP such as lack of professional autonomy to change practice due to resistance from organisational authority. ${ }^{[12,17,18]}$ In addition, inherent bureaucracies in some healthcare systems have been found to impede EBP implementation. ${ }^{[17]}$ In Nigeria, for example, the current healthcare workforce is traditionally bureaucratised and does not embrace principles promoting improvement in care quality. ${ }^{[19]}$ Bureaucracy is an administrative method that is characterised by division of labour, rules, regulations, hierarchy of authority, technical competence, among others. ${ }^{[20]}$ Bureaucracy is a useful management tool which aims to achieve effective management of large organisations (e.g. hospitals) and foster accountability among professionals. ${ }^{[20]}$ However, bureaucracy has enormous demerits and may impede EBP implementation in healthcare organisations that are founded on bureaucratic norms.

Ideally, nurse managers should be positioned to create environment that is supportive of EBP since they play major roles in establishing unit cultures that shape practices. ${ }^{[13,21]}$ However, nurse managers do need to be supported by organisational policy that encourages active participation in implementation processes. ${ }^{[5,16]}$ As bureaucracy generates top-down management approach, nurses and nurse managers

Published by Sciedu Press may not have significant influence in overcoming barriers related to decision-making, resource or budgetary control. Therefore, nurse managers will need to rely on the influences and power of those in authority to steer implementation of new ideas such as EBP. ${ }^{[20]}$ Certainly, these sorts of circumstances will further complicate the struggle to implement and sustain EBP. Against this background, this study explored experiences of nurses implementing EBP in a bureaucratic acute care setting in Nigeria.

\section{Methods}

\subsection{Research design}

An exploratory qualitative approach was adopted in this study. In-depth, face-to-face interviews was utilised to gather data from participants. A qualitative approach offers the fluidity required in exploring social actions due to its naturalistic nature, ${ }^{[22]}$ thereby enabling understanding of day-to-day activities of individuals, groups or organisations. ${ }^{[23]}$ Since nursing practices as well as EBPs occur within the natural clinical setting where individuals, for example, nurses and patients interact, it was envisaged that these interactions may have been shaped by prior experiences, cultures, values and interests.

\subsection{Setting}

The setting for this study was a 540 bedded Teaching Hospital that provided a range of health services to diverse population groups within a state capital, in Nigeria. EBP implementation is likely to be influenced by several structural, procedural and inter-professional dynamics occurring within the acute care setting as it is highly characterised by high rate of uncertainties. Our intention was to explore EBP in a complex and challenging clinical environment where rich information was likely available. Therefore, medical and surgical wards were purposively selected as a range of diverse clinical conditions were being managed there-in.

\subsection{Sample}

Sample for this study consisted of 12 staff nurses (SN) and 11 ward managers (WM) who have worked in their roles for at least a year. These participants were physically approached at the study sites and purposively recruited to participate in the study. Purposive sampling technique was based on practicality as the hospital is in a geographical area that facilitated its access. Overall, a total of 23 participants who gave informed consent were interviewed.

\subsection{Ethical consideration}

Ethical approval was granted by the Research Ethics Committee of the hospital (REC protocol ID: 1105/2015, 1908/2015). Research ethics govern the standards of conduct for scientific 
researchers, and as such it is important to adhere to ethical principles in order to protect the dignity, rights and welfare of research participants. ${ }^{[24]}$ Participants were recruited between June and September 2015 and were provided with information sheet outlining the study's purpose, their involvement as well as strategies for maintaining confidentiality and anonymity. Participants were fully informed about their rights to withdraw at any time, if data analysis has not been completed.

\subsection{Data collection}

Data collection for this study took place between September 2015 and April 2016. Data was gathered through in-depth, face-to-face interviews with participants using an open approach that allowed participants freedom to share their perceptions, experiences and feelings. At the beginning of interview sessions, participants were required to complete demographic questionnaires that focused on age, gender, years of experience and degree attained. In addition, key concepts, for example, EBP were first explained to all 23 participants during recruitment to ensure clarity prior to commencing interviews. An interview guide developed by the authors was first pilot tested with 2 participants and was reviewed and amended appropriately, prior to interviewing the remaining 21 participants. The interview guide consisted of one broad question and a list of several sub-questions or prompts (see Appendix 1 for interview guide). This was to allow participants the flexibility to talk through their areas of interest and capture broadest perspectives of how they constructed their experiences of implementing EBP. Prompts (see Appendix 1) were used to explore further clarifications about issues of relevance. Participants who had further comments were allowed the opportunity to include them prior to ending interview sessions. Interviews were conducted by the lead author with each session lasting for 50-90 minutes and were digitally recorded to ensure accuracy. All interview sessions took place at a time that was convenient to participants and in quite rooms, most of which were ward offices.

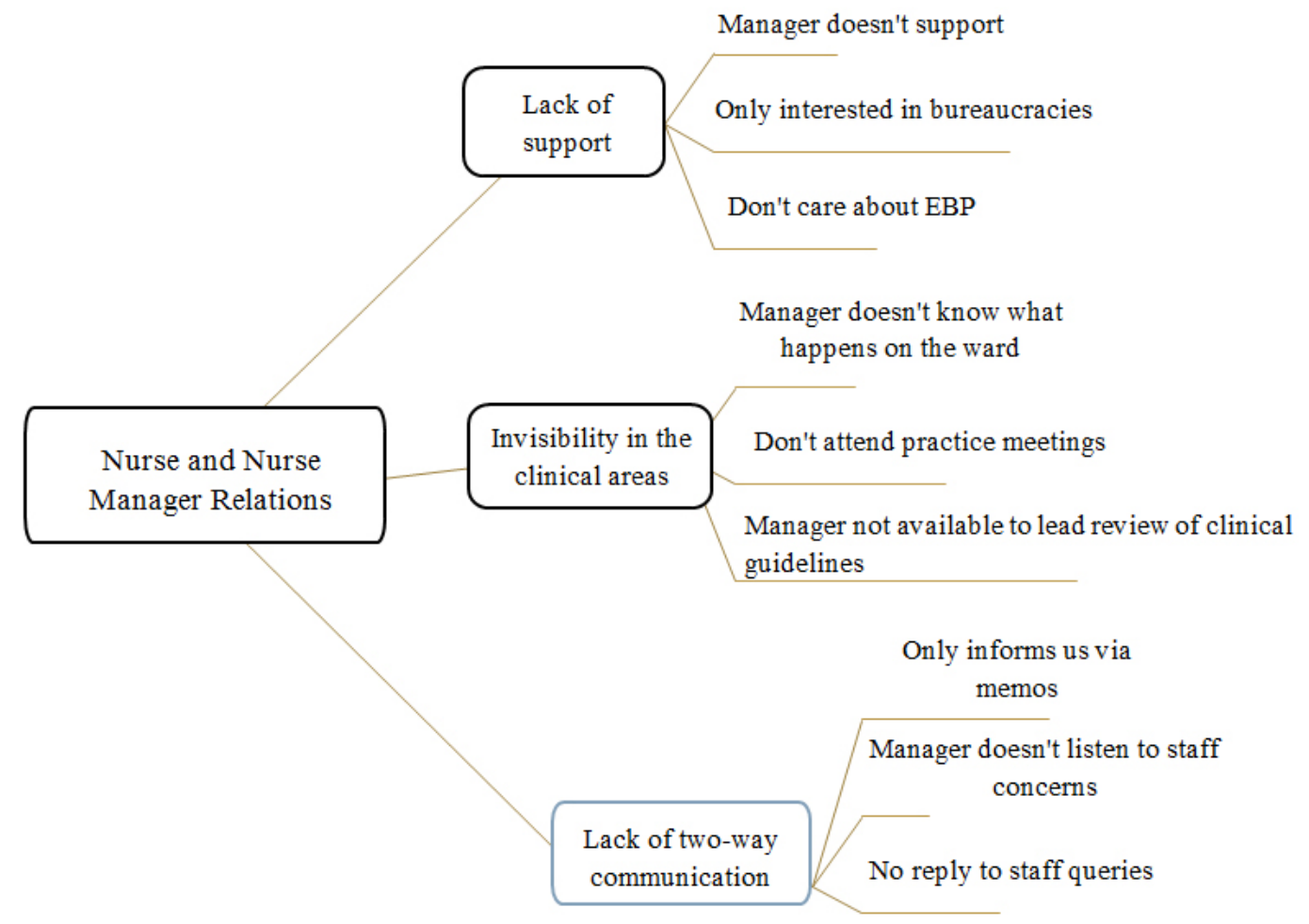

Figure 1. Example of data categorisation that generated codes which yielded initial thematic map

\subsection{Method of data analysis}

Thematic analysis approach was considered suitable and was utilised to analyse the study's data. Thematic analysis offers a strategy for organising and interpreting data to produce a detailed account of, and in-depth understanding of experiences. ${ }^{[25]}$ Thematic analysis was utilised to analyse data. ${ }^{[25]}$ Firstly, to gain familiarisation with data, two of the authors listened to interview, and transcribed them. Following interview transcription, transcripts (176 pages) were reviewed by two other authors. The second stage of analysis involved the creation of codes which generated initial categories. Data was coded using NVivo version 11 by the lead author and was reviewed afterwards by the three other authors. ${ }^{[26]}$ The third phase of data analysis entailed the collation and categorisa- 
tion of generated codes which yielded initial thematic maps (see Figure 1). These initial thematic maps were refined, retained or collapsed. The final phase yielded a satisfactory thematic map which were defined and retained as main themes, which are reported as findings. Overall, data analysis was iterative and involved use of memo to capture analytic thoughts, feelings and insights about the data.

\subsection{Ensuring research rigour}

Establishing rigour in research entails considering credibility, transferability and dependability. ${ }^{[10,27]}$ Credibility refers to ways in which data are interpreted. ${ }^{[28]}$ Although qualitative research is an interpretive process, interpretations may need to be substantiated or supported in some way. We essentially embedded reflexivity in all our considerations by evaluating our potential impact on the research process. While reflexivity does not completely control bias it provides account of actions taken to minimise it. We acknowledge that my professional background as nurses provided insights and interest into the phenomenon investigated and might have had an impact on how we interpreted the data. To maintain credibility and achieve a consensus, all the authors were involved in analysis of data until the final report was completed. Transferability involves providing the reader with sufficient information about the study setting. ${ }^{[22]}$ We have provided a summary of all relevant information about the setting in which the study was conducted. Dependability involves providing sufficient information on both the data collection and data analysis processes to enable the decision-making trail to be followed. ${ }^{[6,19]}$ One of the authors was a novice in qualitative research and was involved in checking dependability by questioning each steps of the process in order to identify how the categories and themes were generated.

\section{Results}

Participants in this study were 5 males and 18 females who were within the age limit of 28-58 years. All of whom had practised for at least 5 years with majority of them having over 15 years of clinical experience. As seen in figure 3.1 below, four key themes were generated revealing how the Nigerian bureaucracy impacts EBPs in nursing. The first theme is "top-down managerial approach" which was derived from two categories: "hierarchical decision-making" and "imposed change". The second theme is "nurse and nurse manager relationship" which was derived from three main categories: "lack of support", "invisibility in the clinical areas", "lack of communication". The third theme is "managerial prerogative" which was derived from two categories: "lack of visibility in the boardroom" and "exclusion from decision-making". The fourth theme is "managerial autonomy" which was derived from "structural positioning" and "lack of managerial authority". These themes will be described and linked to earlier research and literature.

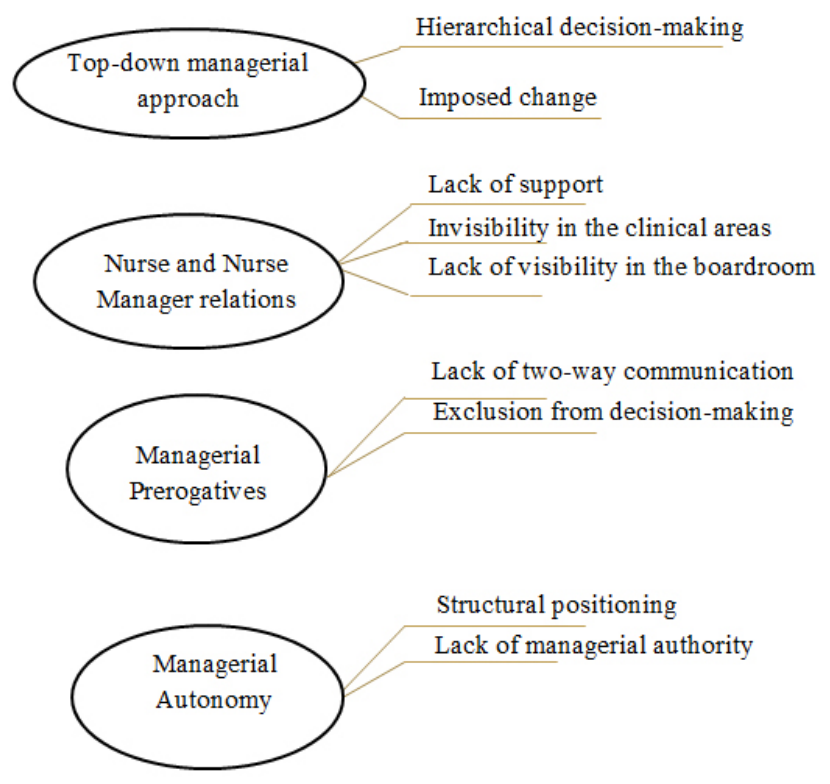

Figure 2. Thematic map

\subsection{Top down managerial approach}

Findings show that the practice setting was bureaucratically structured with key individuals hierarchically positioned. Strategic managerial decisions were top down driven and largely undemocratic as hospital authority has continuously exercised control over goals with nurse managers being were required to comply with decisions made at the upper level. Nurses were required to focus on achieving maximum throughputs rather than engage in activities promoting EBP. There were conflicts between what nurses were required to do in practice and how they desired to deliver nursing practice. As seen in the quotes below, some of the nurses perceived that they were not supported in developing new ideas that would have promoted delivery of EBP.

“... it's been long I wanted to introduce some strategies that would help us evidence-based practice, but the management preferred that we [nurses] focus on facilitating patient journey. .. as a nurse manager I was highly interested in EBP and I initiated the process and created a team for it but I was shunned by the management..." (WM, 02)

“... the management prefers that we focus on admissions and discharges rather than embark on evidence-based practice or even research projects because they cost money... " (SN, 09)

These perceptions depict a situation where nurses did not have the authority to determine what they did in practice. While it seems that nurses have genuine strategies for deliv- 
ering EBP, there are indications that the hospital authority was rather keen on achieving maximum throughputs than implement EBP. This reflects Professional Dominance Theory's conceptualisation of managerialism as an ideology that denies authority to expertise by organising for efficiency and rationally imposing guidance on both choices of consumers as well as productive works of professionals. ${ }^{[29]}$ However, the Ministry of Health which is responsible for controlling the country's healthcare system was said to have developed national clinical practice guidelines (CPGs). These CPGs were being circulated across all hospitals with practitioners, including nurses being mandated to implement them.

"... the ministry of health brought the idea of evidence-based practice with some practice guidelines which they mandated us to implement... although we[nurses] didn't have the opportunity to make inputs regarding implementation we tried to utilise the guidelines but I can't say that it we were successful because of the kind of problems we encountered... " (WM, 01)

“... the guidelines didn't meet the expected standards... a lot of things were wrong with it and we had to do some rechecking, a kind of adaption to enable us to implement them... hopefully, we succeed..." (SN, 07)

"The guidelines need reworking because I've noticed some discrepancies in it... ok when you look at the procedure for treating unconscious patients from admission to time the patient regains consciousness, but it doesn't say what to do afterwards... the same thing with bladder wash out..." (WM, 01)

Bureaucrats from the Ministry of Health and hospitals' management initially negotiated on how to implement these national CPGs without necessarily involving nurse managers. Nurse Managers might not have been involved in the negotiations due to inherent bureaucracies and their lack of visibility at the upper management level. While nurse managers attempted to implement the CGPs, it appears that adequate contextual information regarding how the CGPs were to be implemented was lacking. There were indications that implementation of these CPGs was not successful due to probable lack of fit or adaptability to the local settings. This might suggest that prior plan did not consider relevant requirements needed to achieve successful implementation of the CPGs. Previous studies report that adequate understanding of implementation context is crucial in determining successes of what is to be implemented. ${ }^{[3,13]}$

\subsection{Nurse and nurse manager relations}

The relationships between nurse managers and nursing staff were perceived to be characterised by power relations.
Nurses expressed that nurse managers did not empower them to engage in initiatives promoting EBP. Seemingly, nurses were devoted to delivering changes that would have facilitated EBP in their various wards, however, there were no supplementary support from their nurse managers. As seen in the quotes below, it appears that nurse managers were in part limited by the bureaucratic norms characterising the settings.

"... our manager [nurse manager] is more interested in bureaucracies, but she failed to understand that we can't deliver evidence-based practice without having the necessary support..." (SN, 01)

“... does she even care about evidence-based practice? She doesn't care about what's happening in the ward except if she wants to know the number of patients that have been admitted or discharged in the ward." (SN, 05)

From these perspectives, it does not appear like nurse managers were keen on implementing EBP. Requiring nurses to focus on facilitating admissions and discharges to ensure speedy patient journey, perhaps to generate income since the Nigeria healthcare service operates a pay-for-healthcare system. Therefore, nurse managers might have contributed in forestalling EBP as they were perceived to have focused more on saving their managerial positions working towards meeting organisational targets of achieving maximum throughputs. As seen in the quotes below, nurse managers might have failed to demonstrate clear leadership roles that may have promoted EBP implementation.

“...the nurse manager is meant to lead implementation activities. . how do we deliver evidence-based practice when there are many problems confronting us... I understand that she [nurse manager] may not be able to manoeuvre her way through the top management but at least she can motivate us by her actions." (WM, 06)

The nurse managers may not have been well positioned in way that they could have created a supportive environment that provides evidence-based care. Perhaps due to the hierarchical constraints generated by the inherent bureaucracies in the setting. Previous studies report that lack of managerial support can impede implementation of EBP. ${ }^{[12,30]}$ However, Nurse Managers may have worked towards complying with decisions made by top managers to preserve their jobs which are one of the demerits of bureaucracy.

\subsection{Managerial prerogatives}

Bureaucratic principles enshrined in healthcare management in Nigeria endorse hierarchical managerial structures in public hospitals. This might imply that managerial prerogatives were derivable from positions of individual managers in the 
hospital hierarchy. The authority accruable to individual nurse managers was organically contingent on the administrative structures. Consequently, top managers in these settings were responsible for making key managerial decisions, even as it relates to nursing and EBP implementation.

"If I had my way, I'll develop plans that will enable us to expand evidence-based practice in my ward...on several occasions I have had my proposals thrown back by the management because they can't fund research, so how do we implement evidence-based practice..." (WM, 10)

This might imply that ward managers would deliver EBP if they had the managerial autonomy to initiate strategies for implementation. In the quote above, proposals aiming to develop EBP initiatives remain unfunded, which impedes EBP. The existing top down hierarchy did not allow nurse managers who are at the lower level to decide what they do. Again, nurse managers appear to have been excluded from management meetings where agenda were set, thereby denying them of the opportunity to discuss how they might organise or implement EBP in these settings.

“... they [top management] don't listen to me and it's so frustrating that I can't even discuss evidence-based practice at the management meetings because I'm not even invited to meetings..." (WM, 07)

It is frustrating that nurse managers have not been authoritatively positioned to be involved in administrative decisionmaking. Positioning of WMs in the hierarchy seems to have deprived them authority to plan for EBP. Therefore, their roles might have been limited by the command structure within organisation.

\subsection{Managerial autonomy}

Findings so far discussed raise the question of whether nurse managers had requisite managerial autonomy to drive EBP implementation. As seen in the quotes below, nurses suggest that existing organisational structure compromises the managerial autonomy of nurse managers. Thus, nurse managers have not been properly positioned to autonomously drive EBP in their various units.

“... she [manager] is meant to provide us [nurses] with opportunities to develop strategies that can facilitate evidencebased practice but it's not happening... I also understand that the top management have put her in a very tight position where she doesn't seem to have options.” (WM, 11)

The quote above depicts that nurse managers might have been subjugated to a position where they could only remain submissive to the top managers. While nurse managers were expected to create circumstances that would have facilitated

Published by Sciedu Press
EBP, they acknowledged that nurse managers lacked the managerial autonomy to do so. There were indications that nurse manager's ingenious ideas could not flow from the bottom to the top ladder, since they were hardly given the opportunity to make managerial decisions.

“... I don't think there is much that the nurse manager can do since she doesn't have the authority to make real changes in our practice... we [nurses] can do our best but I'm not sure there is proper ground to expand evidence based practice here in the hospital at this moment." (WM, 03)

There is sense of powerlessness on the part of nursing leadership as seen in the quote above. Existing bureaucracies in these settings might have made it difficult for the nurse managers to affect necessary changes in practice. Inability of the nurse managers to influence rules, regulations and procedures as laid down by the hierarchy might imply that nursing practices have become immune to developmental changes such as EBP implementation.

\section{Discussion}

Findings of this study present complex circumstances around implementation of EBP in an acute care setting. These complexities are contingent on a range of contextual factors, most of which reinforce the position of previous studies recognising relevance of nursing management in EBP implementing processes. ${ }^{[5,17,31]}$ Specifically, findings of this study highlight the importance of organisations and context within which nurses and nurse managers operate, including the components characterising these elements. Finding of this study present a complex picture of four main factors which are intrinsic in a clinical environment where EBP in nursing may be implemented. The crucial need to better understand how organisational contextual factors impact EBP implementation in nursing has been previously reported. ${ }^{[7,16]}$ While evidence emerging from this study support the assumption that EBP implementation is shaped by organisational contextual factors, it further implicates known demerits of organisational bureaucracy in EBP implementation in nursing. The governance of healthcare services in Nigeria is dominated by organisational bureaucracy, which in turn generates top down approaches disempowering nurse managers in their quest to drive EBP. Bureaucracy created environment undermining nurses' innovativeness because they were located at the bottom of the hierarchy. Not only that the top managers did not prioritise EBP, they equally generated hierarchical constraints that consequently placed limits on the nurse's ability to initiate and or implement new ideas. Organisational support for the adoption of EBP may likely be a precondition for its implementation throughout the organisation. Leadership behaviours of nurse managers and those in management 
positions are reported to play key role in successfully implementing evidence in practice. ${ }^{[21]}$ Similarly, hierarchical organisational structure has been reported as limiting nurse manager's ability in driving EBP. ${ }^{[12,31]}$ Findings of this study indicate that nurse manager's structural positioning did not accord them managerial visibility required to influence organisational policies. They did not have much options other than adhere to bureaucratic rules that were enforced by top managers. Consequently, nurses were meant to behave as subordinated employees whose works were subverted as they could not voice out their concerns due to fear, and when they expressed their concerns, they were not listened to by top management.

Top down managerial approaches resulted in a situation where nurse managers were excluded from participating in strategic planning, budgeting and organisational decisionmaking, even on issues concerning nursing practices. This, of course, may have contributed in the non-implementation of the national CPGs that were developed by the Ministry of Health as discussed in the findings. There were indications that the national CGPs were not properly adapted prior to implementation which might have led nurses to consider it as an imposed change. Top down is interlinked with failed implementation processes and can impeded innovativeness within the healthcare setting ${ }^{[32]}$ just as drawbacks exist when evidence (practice guidelines) is incompatible with the local context where implementation occurs. ${ }^{[1]}$ Adequate understanding of the context of implementation is crucial in determining successes of what is to be implemented. ${ }^{[7,26]}$ Seemingly, planning regarding implementation of the national CPGs did not consider relevant requirements before setting out as reflected in the failed efforts despite nurses attempting to implement them.

Nurses and nurse managers did not achieve or maintain a cordial relationship. Their relationship was marred with lack of communication, lack of mentoring, infrequent face to face meetings, lack of forum for knowledge exchange, lack of collaborative and innovative problem solving as well as lack of support for effort aiming to raise new change in practice ideas. Nurses consistently expressed that nurse managers were not interested in patient care activities as they were not visible in various wards. Consequently, it was disempowering for nurses to work without the support of their own managers. There is an inter-link between empowered work environment as well as quality of care. ${ }^{[1,11,21]}$ Nurse Managers who were empowered managed to drive change in practices as they could mobilise internal resources, support as well as quality assurance structures critical to EBP implementation. ${ }^{[9,16]}$
Inherent hierarchical structure in these settings implied that nurses were not positioned in a way that could have afforded them visibility to challenge unfavourable policies. They did not have options other than adhere to bureaucratic rules that were enforced through disciplinary power. Therefore, nurses' behaviours as well as actions were largely shaped by managerial practices and power imbalances, promoting nursing subordination. Nurse Managers could not voice out their concerns due to fear, and when they expressed their concerns, they were not listened to by top management. Previous studies report that lack of administrative as well as leadership supports hinders implementation of EBP in nursing. ${ }^{[30,33,34]}$ Nurse Managers are meant to drive practice changes by disseminating, synthesising, and spreading information about new innovations whilst mediating strategies for implementing EBP.

\section{Strengths and limitations}

Findings of this study can be considered to have cast light on the experiences of nurses implementing EBPs in a bureaucratic environment and may be judged in relation to the Nigerian clinical environment or other similar settings. However, it may be a limitation that this study has only explored perspectives of staff nurses and ward managers and have not necessarily involved patients and the hospital authority. However, involving patients may not have necessarily changed the information as they are not experts in EBP implementation.

\section{CONCLUSIONS AND IMPLICATIONS}

Findings reflect complexities of implementing EBP in a bureaucratic healthcare setting. There are indications that bureaucracy can deny nurses professional and managerial autonomy needed in driving EBPs in nursing practice. There were indications that nurses were managed through bureaucratic rules, and in a setting where fiscal and hierarchical constraints place limits on nurse's autonomy to initiate or implement EBP. Nurses, including nurse managers did not have prerogatives to allocate resources, and, so, have failed to specifically prioritise EBP projects within the settings. Nurse Managers had limited authority and have not generally participated in key governance activities such as strategic planning, budgeting as well as wider organisational decision-making. In sum, EBP was impeded by strict focus on productivity, inefficient staffing, increased workload, and lack of funding for EBP related activities, rejection of nurses' proposals to initiate practice change innovations, lack of managerial interest in EBP. Currently, nursing practices in these settings appear to be traditionally based on rituals and routine which cannot meet the contemporary patient's healthcare needs. There is need for nurses to have greater involvement 
in healthcare management. There is need for nurse managers to have adequate managerial visibility that can afford them greater influence and impetus to engage in EBP implementation. Nurse Managers requires empowerment to create opportunities for EBPs.

\section{CONFlicts of InTEREST Disclosure}

The authors declare they have no conflicts of interest.

\section{REFERENCES}

[1] Melnyk BM, Gallagher-Ford L, Zellefrow C, et al. The first U.S. study on nurses' evidence-based practice competencies indicates major deficits that threaten healthcare quality, safety and patient outcomes. Worldviews on Evidence-Based Nursing. 2018; 15(1): 16-25. PMid: 29278664. https://doi.org/10.1111/wvn.12269

[2] Ominyi J. Exploring the influence of power dynamics on the evidencebased practice implementation in clinical nursing: a case of the Nigerian acute care setting. International Journal of Current Research. 2019; 11(02): 1175-1180.

[3] Rycroft-Malone J, Seers K, Chandler J, et al. The role of evidence, context and facilitation in an implementation trial; implications for the development of the PARIHS framework. Implementation Science. 2013; 8: 28. PMid: 23497438. https ://doi.org/10.1186/1748 $-5908-8-28$

[4] Sackett DL, Straus SE, Richardson WS, et al. Evidence-based medicine: How to practice and teach. EBM (2 ed.). New York: Churchill Livingstone; 2000.

[5] Melnyk BM, Gallagher-Ford L, Thomas BK, et al. A study of chief nurse executives indicates low prioritization of evidence-based practice and shortcomings in hospital performance metrics across the United States. Worldviews on Evidence-Based Nursing. 2016; 13(1): 6-14. PMid: 26873371. https://doi .org/10.1111/wvn. 12133

[6] Shayah SJ, Kiwanuka F, Nakaye Z. Barriers associated with evidencebased practice among nurses in low and middle-income countries: a systematic review. Worldviews on Evidence-Based Nursing. 2019; 16(1): 12-20. PMid: 30604471. https://doi .org/10.1111/wvn. 12337

[7] Melnyk BM, Fineout-Overholt E, Giggleman M, et al. Correlates among cognitive beliefs, EBP implementation, organisational culture, cohesion and job satisfaction in evidence-based practice mentors from a community hospital system. Nursing Outlook. 2010; 58: 301308. PMid: 21074647 . https://doi.org/10.1016/j. outlook. 2010.06 .002

[8] Sadeghi-Bazargani H, Sadegh-Tabrizi J, Azami-Aghdash S. Barriers to evidence-based medicine: a systematic review. Journal of Evaluation in Clinical Practice. 2014; 20: 793-80. PMid: 25130323. https://doi.org/10.1111/jep.12222

[9] Sandstrom B, Borglin G, Nilson R, et al. promoting the implementation of evidence-based practice: a literature review focusing on the role of nursing leadership. Evidence Nursing. 2011; 8(4): 212223. PMid: 21401858. https://doi.org/10.1111/j.1741-6 787.2011.00216.x

[10] Melnyk B, Fineout-Overholt E, Gallagher-Ford L, et al. The state of evidence-based practice among U.S. nurses. Journal of Nursing Administration. 2012; 42(9): 410-417. PMid: 22922750. https: //doi.org/10.1097/NNA.0b013e3182664e0a

[11] Wilkinson JE, Nutley SM, Davies HTO. An Exploration of the Roles of Nurse Managers in Evidence- Based Practice Implementation. Worldviews on Evidence-Based Nursing. 2011; 8(4): 228-236. PMid: 21668735. https://doi.org/10.1111/j.1741-6787.2011.0 $0225 \cdot \mathrm{x}$

Published by Sciedu Press
[12] Ominyi J, Ezeruigbo CFS. How nurse manager's position in the hospital hierarchy influences evidence-based practice implementation in nursing: a qualitative case study of the Nigerian acute care setting. Journal of Nursing Education and Practice. 2019; 9(6): 14-22. https://doi.org/10.5430/jnep.v9n6p14

[13] Ong BO, Morden A, Brooks L, et al. Changing policy and practice: Making sense of national guidelines for osteoarthritis. Social Science and Medicine. 2014; 106: 101-109. PMid: 24556289. https://doi.org/10.1016/j.socscimed.2014.01.036

[14] Wallen G, Mitchell S, Melnyk B, et al. Implementing evidencebased practice: effectiveness of a structured multifaceted mentorship programme. Journal of Advanced Nursing. 2010; 66(12): 27612771. PMid: 20825512. https://doi.org/10.1111/j.1365-2 $648.2010 .05442 . x$

[15] Holloway I. Qualitative Research in Health Care. Open University Press; 2008.

[16] Kueny A, Shever LL, Mackin ML, et al. Facilitating the implementation of evidence-based practice through contextual support and nursing leadership. Journal of Healthcare Leadership. 2015; 7: 29-39. PMid: 29355177. https://doi.org/10.2147/JHL.S45077

[17] Cheng L, Broome ME, Feng S, et al. Factors influencing implementation of evidence in Chinese nursing practice. Journal of Clinical Nursing. 2017; 26: 5103-5112. PMid: 28833737. https: //doi.org/10.1111/jocn.14053

[18] Grove A, Clarke A, Currie G. the barriers and facilitators to the implementation of clinical guidance in elective orthopaedic surgery: a qualitative study protocol. Implementation Science. 2015: 10: 81. PMid: 26033075. https://doi.org/10.1186/s13012-015-0273-6

[19] Alubo O, Hunduh V. Medical dominance and resistance in Nigeria's health care systems. International Journal of Health Services. 2017; 47(4): 778-794. PMid: 27793984. https://doi.org/10.1177/ 0020731416675981

[20] Andreasson J, Ljungar E, Ahlstrom L, et al. Professional bureaucracy and health care manager's planned change strategies: governance in Swedish healthcare. Nordic Journal of Working Life Studies. 2018; 8(1): 23-41. https://doi.org/10.18291/njwls.v8i1. 104849

[21] Cheryl AF, Sheeron J. Creating a culture of EBP: What's a manager to do? Nursing Management. 2014; 10: 21-23. PMid: 25244237. ht tps://doi.org/10.1097/01. NUMA.0000453943.62534.03

[22] Davies MB, Hughes N. Doing a successful research project: Using qualitative or quantitative methods. Palgrave: Macmillan; 2014

[23] Melnyk BM, Fineout-Overholt E. Evidence-Based Practice in Nursing and Healthcare: A Guide to Best Practice. 2nd ed. Lip- pincott, Williams \& Wilkins, Philadelphia, PA; 2011.

[24] World Health Organisation. Ethical standards and procedures for research with human beings. 2018. Available from: https://www . who. int/ethics/research. Accessed Friday September 2019.

[25] Braun V, Clarke V. Using thematic analysis in psychology. Qualitative Research in Psychology. 2006; 3(2): 77-101. https://doi.or g/10.1191/1478088706qp063oa 
[26] QSR International. NVivo 11. Washington, USA: QSR International; 2012.

[27] Gold R. Roles in sociological field observation. Social Forces. 1958; 36: 217-213. https://doi.org/10.2307/2573808

[28] Crowe M, Inder M, Porter R. Conducting qualitative research in mental health: Thematic and content analyses. Australian \& New Zealand Journal of Psychiatry. 2015; 49(7): 616-623. PMid: 25900973. https://doi.org/10.1177/0004867415582053

[29] Freidson E. Professionalism: the third logic. Cambridge: Polity Press; 2001.

[30] Baatiema L, Otim ME, Mnatzaganian G, et al. Health professionals' views on the barriers and enablers to evidence- based practice for acute stroke care: A systematic review. Implementation Science. 2018; 12(1): 74. PMid: 28583164. https://doi.org/10.1186/ s13012-017-0599-3
[31] Heydari A, Zeydi AE. Nurses' Perceptions and Barriers for Adoption of Evidence Based Practice in Primary Care: Bridging the Gap. Journal of Caring Science. 2014; 3(4): 265-275.

[32] Andersson AE, Gifford G, Nilsson K. Improving care in surgery - a qualitative study of managers' experiences of implementing evidencebased practice in the operating room. Journal of Hospital Administration. 2015; 4(4): 73-83. https ://doi.org/10.5430/jha.v4n4 p73

[33] Brown C, Wickline M, Ecoff L, et al. Nursing practice, knowledge, attitudes and perceived barriers to evidence-based practice at an academic medical centre. Journal of Advanced Nursing. 2009; 65(2): 371-381. PMid: 19040688. https://doi.org/10.1111/j.1365 $-2648.2008 .04878 . x$

[34] Sin MK, Bliquez R. Teaching evidence-based practice to undergraduate nursing students. Journal of Professional Nursing. 2017; 33: 447-451. PMid: 29157574. https ://doi.org/10.1016/j . prof nurs.2017.06.003 